\title{
Rainbow spanning subgraphs of small diameter in edge-colored complete graphs
}

\author{
Sogol Jahanbekam*and Douglas B. West ${ }^{\dagger}$
}

Revised March 2015

\begin{abstract}
Let $s(n, t)$ be the maximum number of colors in an edge-coloring of the complete graph $K_{n}$ that has no rainbow spanning subgraph with diameter at most $t$. We prove $s(n, t)=\left(\begin{array}{c}n-2 \\ 2\end{array}\right)+1$ for $n, t \geq 3$, while $s(n, 2)=\left(\begin{array}{c}n-2 \\ 2\end{array}\right)+\left\lfloor\frac{n-1}{2}\right\rfloor$ for $n \neq 4($ and $s(4,2)=2)$.
\end{abstract} Keywords: spanning subgraph, rainbow subgraph, diameter, anti-Ramsey number $M S C$ code: $05 \mathrm{C} 55,05 \mathrm{C} 35$

\section{Introduction}

A rainbow subgraph of an edge-colored graph $G$ is a subgraph whose edges have distinct colors. The general anti-Ramsey problem asks for the maximum number of colors in an edge-coloring of $K_{n}$ having no rainbow copy of some graph in a class $\mathcal{F}$; this maximum number of colors is the anti-Ramsey number $\operatorname{AR}(n, \mathcal{F})$. (The Ramsey problem can be interpreted as asking for the minimum number of colors in an edge-coloring having no monochromatic copy of a graph in $\mathcal{F}$.) Early results considered the problem with $\mathcal{F}$ being a single graph: see [1] for a survey and [4] for the notable determination of $\operatorname{AR}\left(n, C_{k}\right)$. More recent work considers problems where $\mathcal{F}$ consists of spanning subgraphs of $K_{n}$; see [2] for a discussion of such problems involving spanning cycles, perfect matchings, and spanning trees.

In this paper, we study when rainbow spanning subgraphs of small diameter are forced. Let $s(n, t)$ be the maximum number of colors in an edge-coloring of $K_{n}$ not having a rainbow spanning subgraph with diameter at most $t$. We compute $s(n, t)$. Previously, Montágh [3] obtained an upper bound on $s(n, 3)$, showing that $\operatorname{AR}\left(n, \mathcal{D}_{n}\right)=\left(\begin{array}{c}n-2 \\ 2\end{array}\right)+1$, where $\mathcal{D}_{n}$ is the

*jahanbe1@illinois.edu; University of Illinois, Urbana, IL. Research supported in part by National Science Foundation grant DMS 09-01276.

†west@math.uiuc.edu; Zhejiang Normal University, Jinhua, China, and University of Illinois, Urbana, IL. Research supported by Recruitment Program of Foreign Experts, 1000 Talent Plan, State Administration of Foreign Experts Affairs, China. 
family of $n$-vertex double-stars whose vertex of second largest degree is at most 4 . That is, rainbow double stars that are "almost" stars are forced by having more than $\left(\begin{array}{c}n-2 \\ 2\end{array}\right)+1$ colors. To prove $s(n, t) \geq\left(\begin{array}{c}n-2 \\ 2\end{array}\right)+1$ when $t \geq 3$, we present an edge-coloring of $K_{n} \operatorname{using}\left(\begin{array}{c}n-2 \\ 2\end{array}\right)+1$ colors that has no rainbow spanning subgraph with diameter at most $t$.

With more colors, such a subgraph is forced. Upper bounds for anti-Ramsey problems generally use the notion of representing subgraph of an edge-colored complete graph, which is a spanning subgraph containing one edge of each color. We give a short proof that any edge-coloring of $K_{n}$ using at least $\left(\begin{array}{c}n-2 \\ 2\end{array}\right)+2$ colors has a representing subgraph with diameter at most 3 . Thus when $t \geq 3$ the answer does not depend on $t$. The answer for the special case $t=2$ is somewhat different.

Theorem 1.1. $s(n, t)=\left(\begin{array}{cl}n-2 \\ 2\end{array}\right)+ \begin{cases}1 & \text { for } n, t \geq 3 \\ 2 & \text { for }(n, t)=(4,2), \\ \left\lfloor\frac{n-1}{2}\right\rfloor & \text { when } t=2 \text { and } n \neq 4 .\end{cases}$

The aim is to show that when too many colors are used, every edge-coloring contains some representing subgraph having the forbidden property.

As noted by a referee, our work suggests the corresponding Turán-type extremal question: How many edges can an $n$-vertex graph have without having a spanning subgraph with diameter at most $t$ ? The answer is $\left(\begin{array}{c}n-1 \\ 2\end{array}\right)$, achieved by $K_{n-1}+K_{1}$.

\section{Constructions and easy cases}

For a vertex $v$ in a graph $G$, let $N_{G}(v)$ be the set of neighbors of $v$, with $N_{G}[v]=N_{G}(v) \cup\{v\}$, and let the degree $d_{G}(v)$ of $v$ be $\left|N_{G}(v)\right|$. An $x, y$-path in $G$ is a path with endpoints $x$ and $y$. The distance $d_{G}(x, y)$ between vertices $x$ and $y$ is the minimum length of an $x, y$-path in $G$, and the diameter $\operatorname{diam}(G)$ is $\max _{x, y \in V(G)} d_{G}(x, y)$. By convention, $d_{G}(x, y)$ is infinite when $G$ has no $x, y$-path. When only one graph is under discussion, it is common to drop the subscripts in $N_{G}(v), N_{G}[v], d_{G}(v)$, and $d_{G}(x, y)$.

Determining $s(n, t)$ is easy when $t \geq 3$.

Lemma 2.1. A connected $n$-vertex graph with at least $\left(\begin{array}{c}n-2 \\ 2\end{array}\right)+2$ edges has diameter at most 3. An n-vertex graph with at least $\left(\begin{array}{c}n-1 \\ 2\end{array}\right)+1$ edges is connected and has diameter at most 2 .

Proof. If $G$ is connected and $\operatorname{diam}(G)>3$, then there exist $x$ and $y$ with $d(x, y)=4$. Let $j=|N(x)|$ and $k=|N(y)|$, and let $l=|V(G)-N[x]-N[y]|$. To avoid having a shorter $x, y$-path, $N(x)$ and $N(y)$ must be disjoint and joined by no edge. Thus $j+k+l=n-2$; also $j, k \geq 1$. Hence $|E(\bar{G})| \geq j+k+2 l+j k+1=n-1+l+j k$. Since $l+j+k=n-2$ and $j, k \geq 1$, the quantity $l+j k$ is minimized when $l=n-4$ and $j=k=1$. Hence $|E(\bar{G})| \geq 2 n-4$, yielding $|E(G)| \leq\left(\begin{array}{c}n-2 \\ 2\end{array}\right)+1$. 
If $\operatorname{diam}(G)>2$, then vertices $x$ and $y$ with $d(x, y)>2$ are nonadjacent and have nonneighbors covering the remaining vertices, so $|E(\bar{G})| \geq n-1$, yielding $|E(G)| \leq\left(\begin{array}{c}n-1 \\ 2\end{array}\right)$.

Lemma 2.2. If $n, t \geq 3$, then $s(n, t)=\left(\begin{array}{c}n-2 \\ 2\end{array}\right)+1$.

Proof. An edge-colored complete graph in which all edges incident to two vertices have the same color has no connected rainbow spanning subgraph. Hence $s(n, t) \geq\left(\begin{array}{c}n-2 \\ 2\end{array}\right)+1$.

For the upper bound, consider a coloring of $E\left(K_{n}\right)$ using at least $\left(\begin{array}{c}n-2 \\ 2\end{array}\right)+2$ colors; we may assume equality. If a representing subgraph $G$ has an isolated vertex, then the other vertices all have degree at least 2 , since otherwise there are at most $\left(\begin{array}{c}n-2 \\ 2\end{array}\right)+1$ edges. Now adding any edge $e$ incident to an isolated vertex $v$ and removing the edge in $G$ with the same color as $e$ yields a representing subgraph with no isolated vertex.

Hence we may assume that $G$ has no isolated vertex. If $G$ is disconnected, then $|E(\bar{G})| \geq$ $2(n-2)$, but $|E(\bar{G})| \leq 2 n-5$. Hence $G$ is connected, and Lemma 2.1 restricts $G$ to diameter at most 3 , as desired.

The difficult case is $t=2$, where we must force a rainbow spanning subgraph with diameter 2 . For $s(3,2)$, two colors are clearly both necessary and sufficient.

Lemma 2.3. $s(4,2)=3$.

Proof. For the lower bound, give colors 1 and 2 to two independent edges, and give color 3 to the remaining 4-cycle. The only spanning subgraph with three edges and diameter 2 is a star, but the coloring has no rainbow star. For the upper bound, every 4-vertex graph with at least four edges has diameter at most 2 .

For $t=2$ with $n>4$, we first provide the construction for the lower bound.

Lemma 2.4. If $n>4$, then $s(n, 2) \geq\left(\begin{array}{c}n-2 \\ 2\end{array}\right)+\left\lfloor\frac{n-1}{2}\right\rfloor$.

Proof. In $K_{n}$, give one color to all edges incident to one vertex $v$ and also to $\left\lceil\frac{n-1}{2}\right\rceil$ edges covering the remaining $n-1$ vertices. Give distinct other colors to the remaining edges. The total number of colors is $\left(\begin{array}{c}n-1 \\ 2\end{array}\right)-\left\lceil\frac{n-1}{2}\right\rceil+1$, which equals $\left(\begin{array}{c}n-2 \\ 2\end{array}\right)+\left\lfloor\frac{n-1}{2}\right\rfloor$. In any rainbow subgraph $G$, the degree of $v$ is at most 1 . Every vertex other than $v$ has two incident edges of the same color and hence has degree at most $n-2$ in $G$. Therefore, not all vertices can be within distance 2 of $v$.

\section{Rainbow subgraphs with diameter 2}

The upper bound for $n>4$ when $t=2$ is the difficult part. Let $\Gamma(v)$ denote the set of edges incident to $v$ in $G$. Say that a color is incident to a vertex $v$ if it is used on an edge in $\Gamma(v)$. A clique in a graph is a set of pairwise adjacent vertices. 
Lemma 3.1. If $n>4$, then $s(n, 2) \leq\left(\begin{array}{c}n-2 \\ 2\end{array}\right)+\left\lfloor\frac{n-1}{2}\right\rfloor$.

Proof. It suffices to study an edge-colored copy $H$ of $K_{n}$ using exactly $\left(\begin{array}{c}n-2 \\ 2\end{array}\right)+\left\lfloor\frac{n-1}{2}\right\rfloor+1$ colors. Suppose that $H$ has no rainbow spanning subgraph with diameter 2. Thus a representing subgraph of $H$ has no spanning complete bipartite subgraph (no copy of $K_{1, n-1}$ or $K_{2, n-2}$ ), and every vertex is incident to at most $n-2$ colors.

Given a vertex $u$, let $G^{\prime}$ be a subgraph of $H-u$ containing one edge of each color not incident to $u$. Avoiding rainbow spanning stars in $H$ requires $\Delta\left(G^{\prime}\right)<n-2$, and hence $\left|E\left(G^{\prime}\right)\right| \leq\left(\begin{array}{c}n-1 \\ 2\end{array}\right)-\left\lceil\frac{n-1}{2}\right\rceil=\left(\begin{array}{c}n-2 \\ 2\end{array}\right)+\left\lfloor\frac{n-1}{2}\right\rfloor-1$. Therefore, at least two colors are incident to $u$. If equality holds, then $\overline{G^{\prime}}$ is 1 -regular (except for one vertex $z$ of degree 2 when $n-1$ is odd). Hence $\overline{G^{\prime}}$ is disconnected (since $n>4$ ), which yields $\operatorname{diam}\left(G^{\prime}\right)=2$. Choose $u v, u w \in \Gamma(u)$ with distinct colors so that either $n$ is even or $\{v, w\} \neq N_{\overline{G^{\prime}}}(z)$. Since $V(H)-\{u, v, w\} \subseteq N_{G^{\prime}}(v) \cup N_{G^{\prime}}(w)$, adding $u v$ and $u w$ to $G^{\prime}$ completes a rainbow spanning subgraph of $H$ with diameter 2. We may therefore assume that every vertex has at least three incident colors.

Let $G$ be any representing subgraph of $H$. Since $\operatorname{diam}(G)>2$, we have $\Delta(G) \leq n-2$. Since $K_{2, n-2} \not \subseteq G$, the vertices with degree $n-2$ form a clique. Pick $y, z \in V(G)$ such that $d_{G}(y, z)=3$, which requires $d_{G}(y)+d_{G}(z) \leq n-2$. Let $a=n-2-\left(d_{G}(y)+d_{G}(z)\right)$. Since $|E(G)|=\left(\begin{array}{c}n-2 \\ 2\end{array}\right)+\left\lfloor\frac{n-1}{2}\right\rfloor+1$,

$$
\sum_{w \notin\{y, z\}} d_{G}(w)=2|E(G)|-(n-2-a)=(n-2)(n-3)+(a+b),
$$

where $b=2$ for even $n$ and $b=3$ for odd $n$. Thus $|X| \geq a+b$, where $X$ is the set of vertices with degree $n-2$ in $G$, with equality only if all vertices outside $X \cup\{y, z\}$ have degree $n-3$. Indeed, with $W=V(G)-X-\{y, z\}$, we have $|X|=a+b+c$, where $c=|W|(n-3)-\sum_{w \in W} d_{G}(w)$. Since $a, c \geq 0$, we have $|X| \geq 2$. In particular, we have proved that if $H$ has no rainbow spanning subgraph with diameter 2, then every representing subgraph of $H$ has at least $b$ vertices of degree $n-2$.

Suppose $\delta(G) \leq 1$. Choose $u, v \in V(G)$ with $u$ having smallest degree and $v$ smallest among the others. Since $|E(G)|=\left(\begin{array}{c}n-2 \\ 2\end{array}\right)+\left\lfloor\frac{n+1}{2}\right\rfloor$, at least $\left\lfloor\frac{n+1}{2}\right\rfloor$ edges are incident to $\{u, v\}$ in $G$, with equality only when $G-\{u, v\}$ is complete. Recall that in $H$ at least three colors are incident to $u$. If all vertices other than $u$ have degree at least 3 in $G$ (which includes all cases with $n>6$ ), then we can bring in an edge of another color at $u$ in place of the edge of that color in $G$, and this will increase the minimum degree. The remaining case is $n \in\{5,6\}$ with three edges incident to $\{u, v\}$ in $G$ and $G-\{u, v\}$ being a complete graph. Again we can increase the minimum degree, since the three edges incident to $\{u, v\}$ can be chosen to be $u v$ and edges of distinct other colors at $u$ and $v$.

Hence we may choose a representing subgraph $G$ with $\delta(G) \geq 2$. Define $y, z, X, W, a, b, c$ as before. Since $d_{G}(y, z)=3$, each vertex of $X$ has $y$ or $z$ as its only nonneighbor and is 
adjacent to all of $W$; hence $\{y, z\}$ is the only pair with no common neighbor. By symmetry, we may assume $\left|X \cap N_{G}(z)\right| \geq\left|X \cap N_{G}(y)\right|$. Choose $x \in X \cap N_{G}(z)$. The color of $x y$ in $H$ appears on an edge $x w$ in $G$, since otherwise $H$ has a spanning rainbow star with center $x$. Let $G^{\prime}=G-x w+x y$. If $w \neq z$, then $\operatorname{diam}\left(G^{\prime}\right)=2$; hence we may assume $w=z$. The exchange reduces the degree only at $z$; if $d_{G}(z) \geq 3$, then $\delta\left(G^{\prime}\right) \geq 2$.

Let $k_{G}=\left|X \cap N_{G}(z)\right|-\left|X \cap N_{G}(y)\right|$. We have $k_{G^{\prime}}<k_{G}$ if $k_{G} \geq 2$. Hence we may assume $k_{G} \in\{0,1\}$ or $d_{G}(z)=2=k_{G}$.

If $d_{G}(z)=2=k_{G}$, then $|X|=2$ and $N_{G}(z)=X$, and the color on $x_{i} y$ appears also on $x_{i} z$, where $X=\left\{x_{1}, x_{2}\right\}$. Now $|X|=a+b+c$ and $b \geq 2$ requires $a=c=0$ and $b=2$. With $a=n-2-\left(d_{G}(y)+d_{G}(z)\right)$ and $d_{G}(z)=2$, we also have $N_{G}(y)=W$. Let $e$ be the edge in $G$ having the same color as $y z$. In $G$, every vertex except $y$ neighbors both vertices of $X$, so every nonadjacent pair not involving $y$ has at least two common neighbors. Hence if $e$ is not incident to $y$, then $\operatorname{diam}(G-e+z y)=2$. If $e=w y$ for some $w \in W$, then let $G^{\prime}=G-\left\{x_{1} z, w y\right\}+\left\{x_{1} y, y z\right\}$, having the same colors as $G$. At each vertex, at most one edge was removed, so it suffices to check that $d_{G^{\prime}}(y, v) \leq 2$ for all $v$. Since $y$ reaches $z$ directly and all other vertices via $x_{1}$, we have $\operatorname{diam}\left(G^{\prime}\right)=2$.

We may therefore assume both that $\delta(G) \geq 2$ and that $N_{G}(y) \cap X$ and $N_{G}(z) \cap X$ partition $X$ into sets differing in size by at most 1 . Furthermore, since $y$ and $z$ each have a neighbor in $X$, any two vertices other than $\{y, z\}$ have a common neighbor in $X$.

Let $e$ be the edge in $G$ with the same color as $y z$, and let $\hat{G}=G-e+y z$. If $e$ has neither endpoint in $X$, then deleting $e$ yields all pairs except $\{y, z\}$ with a common neighbor, so $\operatorname{diam}(\hat{G})=2$. We may therefore assume that $e$ has an endpoint in $X$. Note that since $\delta(G) \geq 2$ and $a=n-2-\left(d_{G}(y)+d_{G}(z)\right)$, we have $d_{G}(y), d_{G}(z) \leq n-4$.

Case 1: $|X| \geq 4$. From the argument above, $y$ and $z$ each have at least two neighbors in $X$. Hence any pair other than $\{y, z\}$ has at least two common neighbors in $X$. Thus deleting $e$ does not increase their distance above 2 , and $\operatorname{diam}(\hat{G})=2$.

Case 2: $|X|=2$, or $|X|=3$ and $n$ is odd. Since $|X|=b$, we have $a=c=0$. Since $d_{G}(y), d_{G}(z) \leq n-4$, we have $d_{\hat{G}}(y), d_{\hat{G}}(z) \leq n-3$. Since also $e$ has an endpoint in $X$, the representing subgraph $\hat{G}$ has fewer than $b$ vertices of degree $n-2$ (which is forbidden).

Case 3: $|X|=3$ and $n$ is even. Since $b=2$, we have $\{a, c\}=\{0,1\}$. When $n=6$, with $d_{G}(y), d_{G}(z) \geq 2$ we have $a=0$ and hence $c=1$, but then the one vertex of $W$ has degree only 2 , which prevents it from being adjacent to all of $X$. Hence we may assume $n \geq 8$.

Let $x$ be an endpoint of $e$ in $X$. Since $z$ has two neighbors in $X$ and $y$ has one neighbor in $X$, we have $\operatorname{diam}(G-e) \leq 2$ unless $e=x y$. Hence $\operatorname{diam}(\hat{G})>2$ requires $e=x y$, and any pair with distance 3 in $\hat{G}$ involves $y$. Since $d_{G}(y) \geq 2$, still $x$ and $y$ have a common neighbor $w \in W$. Since $y z \in E(\hat{G})$, a vertex $w^{\prime}$ with $d_{\hat{G}}\left(w^{\prime}, y\right)=3$ must lie outside $N_{G}(y) \cup N_{G}(z) \cup\{y, z\}$. If $a=0$, then there is no such vertex. Hence $(a, c)=(1,0)$ and 
there is one vertex $w^{\prime}$ in $W-\left(N_{G}(y) \cup N_{G}(z)\right)$. However, $c=0$ yields $d_{G}\left(w^{\prime}\right)=n-3$. Since $y, z \notin N_{G}\left(w^{\prime}\right)$, we have $w \in N_{G}\left(w^{\prime}\right)$, and hence $\operatorname{diam}(\hat{G})=2$.

With Lemmas 2.2-2.4 and 3.1, the proof of Theorem 1.1 is now complete.

\section{References}

[1] S. Fujita, C. Magnant, and K. Ozeki, Rainbow generalizations of Ramsey theory: a survey. Graphs Combin. 26 (2010), 1-30.

[2] S. Jahanbekam and D. B. West, Anti-Ramsey problems for $t$ edge-disjoint rainbow spanning subgraphs: cycles, matchings, or trees. Submitted, 2014.

[3] B. Montágh, Anti-Ramsey numbers of spanning double stars. Acta Univ. Sapientiae Math. 1 (2009), 21-34.

[4] J. J. Montellano-Ballesteros and V. Neumann-Lara, An anti-Ramsey theorem on cycles, Graphs Combin. 21 (2005), 343-354. 